\title{
Impact of Mobility Models on MMS Routing in Wireless Sensor Networks
}

\author{
M.S.Godwin Premi \\ Research Scholar \\ Sathyabama University \\ Chennai-119, India
}

\author{
K.S.Shaji \\ Professor \\ RIITW, Nagercoil-1 \\ Tamil Nadu, India
}

\begin{abstract}
In this paper, we describe about the performance of different mobility models on MMS Routing. MMS routing is the technique used for large wireless sensor networks where MMS indicates multiple mobile sinks. The mobility of sink is considered in random manner in the basic MMS routing. Here we show the effect of different mobility models in the sink's mobility. From the observations what we got from simulations we decide that wind mobility will be more energy efficient and it is good to be selected for wireless sensor network.
\end{abstract}

\section{General Terms}

Mobility, Routing, Wireless Sensor Networks.

\section{Keywords}

MMS Routing, Sink Mobility, Wireless Sensor Networks.

\section{INTRODUCTION}

Wireless sensor networks are widely used to access the information about the physical world, in real time. Almost all the areas of sciences and engineering noticed the benefits of utilizing sensor networks [14], [15]. Wireless sensor network is a collection of large number of tiny sensor nodes. In real time scenarios sensor nodes like Berkley's smart dust, $\mu$-Adaptive multi-domain power aware sensors are deployed as static network. Since this network is a data centric network every node has to sent the information to the central node. The central node is nothing but the sink node. The next evolution of wireless senor network is mobile wireless sensor networks. Mobile sensor network is designed to handle mobility in all its forms. In this either the sensor nodes or sink nodes will be in mobile or both will be mobile. The mobility makes the network to gather more data. Because in static networks, the mobility of sensors, sink nodes and the monitored phenomenon are totally ignored. Based on the applications different mobility models can be employed and they play a vital role in data collection.

This dynamic nature of mobile wireless sensor networks [1], [2], [8] introduces many challenges like coverage, routing protocols, security and data management. In static sensor networks many problems related to above mentioned challenges are discussed by the researchers. But present few researchers addressed few problems for mobile sensor networks. One of the most important problems is the route stability during mobility. The conventional routing protocols for static sensor networks are to be optimized once we introduce mobility. To study the performance of routing protocols under such conditions, it needs to consider the mobility patterns of entire network.

\section{RELATED WORK}

Mobility models applicable for a wireless sensor network can be broadly classified as memory-less models and memory based models. In memory-less models the mobile node doesn't spent any memory to change their location. But in memory based models the mobile nodes use its previously stored database for its movement. The mobility metrics[13] that differentiate one mobility model from another are velocity, angle, acceleration, co-ordinates of a reference node, distance between nodes, transmission range, degree of spatial dependence, degree of temporal dependence and number of mobile nodes. The classification of mobility models [9], [10], [11], [12] is given in figure 1 .

In the Random Walk Mobility Model, a mobile node moves from its current location to a new location by randomly choosing a direction and speed in which to travel. The new speed and direction are both chosen from pre-defined ranges, [speedmin, speedmax $]$ and $[0,2 \pi]$ respectively. Each movement in the Random Walk Mobility Model occurs in a constant time interval $t$, at the end of which a new direction and speed are calculated. The Random Waypoint Mobility Model includes pause times between changes in direction and/or speed. A mobile node waits by staying in one location for a certain period of time (i.e., a pause time) before moving to the new position. Once this time expires, the mobile node chooses a random destination as well as a speed that is uniformly distributed between [0, speedmax]. It then travels towards the newly chosen destination at the selected speed. Upon arrival, the mobile node takes another break before starting the process again. Random Waypoint Mobility Model is similar to the Random Walk Mobility Model if pause time is zero and $[0$, speedmax $]=$ [speedmin, speedmax].In the Random Direction Mobility Model mobile node choose a random direction in which to travel instead of a random destination. After choosing a random direction, the node travels to the boundary. As soon as the boundary is reached the node stops for a certain period of time, chooses another angular direction (between 0 and 180 degrees) and continues the process. The Gauss-Markov Model works on the basis of previous speed and direction for the current move. The City Section mobility model puts constraints on the movement of a node on a city street grid, constructed of horizontal and vertical streets. Each street on the grid is assigned a speed limit. A mobile node moves along the streets according to the speed limit set for that particular street. In geographic models the node movement is restricted to the pathways in the sensing field. The Manhattan mobility model is used to model the movement of cars or people on a city street grid. 


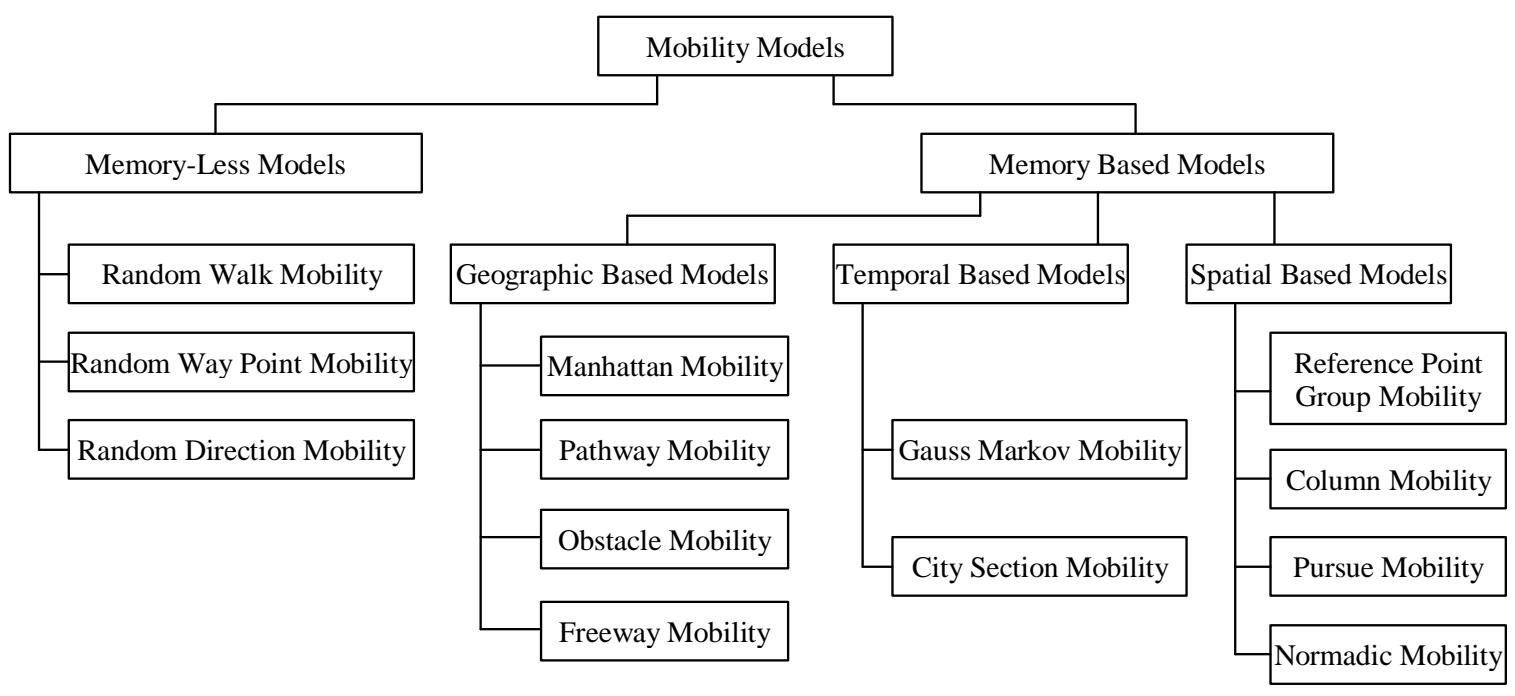

Figure 1: Classifications of Mobility Models

Like the City Section model, each mobile node utilizes a probabilistic approach for movement on the streets. The movement of a node is decided one street at a time. The freeway model is used in exchanging traffic status or tracking a vehicle on a freeway. Reference point group mobility model is widely used in military battlefield communication. In this mode, each group has a logical center called group leader. The group leader motion determines the behavior of group motion. In obstacle mobility model obstacles do affect the movement behavior of mobile nodes.

\section{PROBLEM DESCRIPTION}

\subsection{MMS Routing}

In large wireless sensor networks, in order to handle large number of sensor nodes multiple sink nodes are used. MMS routing is nothing but the Multiple Mobile Sinks routing [5], [6], [7]which is used in large wireless sensor networks. In MMS routing all the sink nodes will collect the data during its stationary position. When they are moved to the new position, each sink node will select the dissemination node. Dissemination node is selected from the available nodes in the coverage area of the sink node [3]. It is selected in such a way that the sensor node which has high available energy. Now the sink node will inform its location to the dissemination node. This dissemination node will inform sink's location to all the sensor nodes by means of flooding technique. Based on the geographic forwarding geocast algorithm [4] the data packets are forwarded from the source sensor node to the sink node because in this geographic algorithm all the nodes know their geographical coordinates. The message flow shown below gives the information about the exchange of messages between the sink node and the sensor nodes in its coverage area. These exchanges of messages will be after the sink node enters into a new position. Every time after the sink changes its location, the location information is updated by means of new dissemination node using flooding technique.

This MMS routing is applicable for the wireless sensor network with static sensor nodes and mobile sink nodes. In the basic MMS routing the random mobility model of the sink node is considered. In this paper we compared different mobility models for the same MMS routing. Every time the change in location of the sink node has to be informed to all the sensor nodes. If the sensor nodes are intelligent enough to know all the eight positions then the energy spent for location update of the sink node by the dissemination node can be completely reduced. Dissemination is not necessary. But if the sink node velocity is changed this will not holds good. So in that case again location update of the sink node is necessary. Because, the sensor nodes do not know at what time the sink node will be available in that particular position.

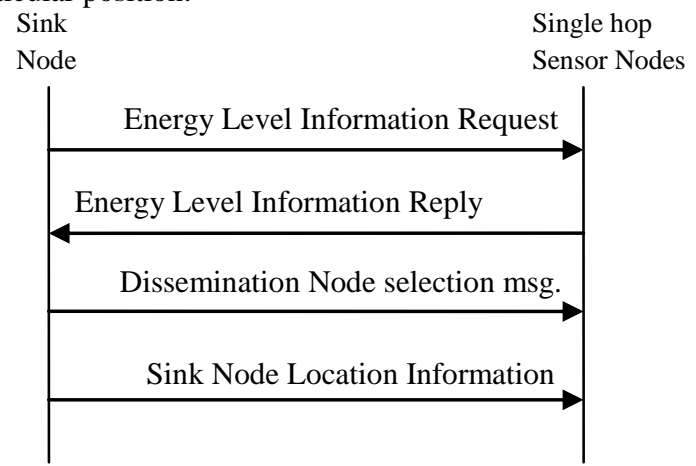

Figure 2: Message flow for the selection of dissemination node

\subsection{Mobility Models}

In wireless sensor network already the nodes have minimum memory. So it is better to select memory less models. But if energy spent for mobility in the overall network can be reduced by spending little bit of memory then we can go for memory based models. The reduction of energy spent for mobility makes more collection of data due to the extension in lifetime. In this paper we applied the mobility model on MMS routing and compared the memory-less model with memory based model and proved that energy is saved. In the memory-less model we selected the random way point mobility in the memory based model we selected geographic based mobility model. Again from the geographic based only circular pathway mobility model is selected. These two mobility models are compared with the wind mobility model which is proposed by us. After analyzing the results of this wind mobility, it is found as good. 


\subsection{Impact of random way point mobility}

In the random way point model the sink node's new position is determined with random velocity and random direction. Figure 3 shows the random way point with only eight static positions or pause times. Actually these static positions are nothing but the pause times in the random way point mobility. Based on this model the time taken to reach each point or position is calculated and then energy spent to reach the positions are also obtained.

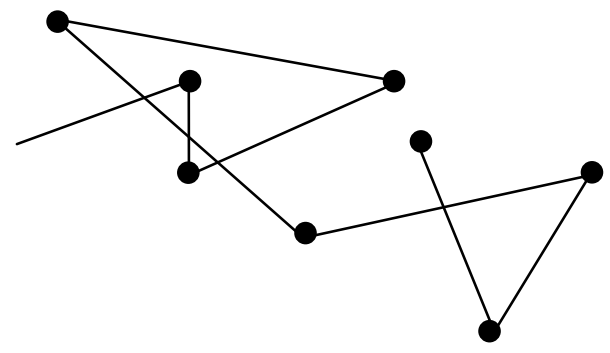

Figure 3: Random way point mobility with 8 point

\subsection{Impact of circular path mobility}

To obtain the impact of geographic based mobility model on MMS routing, the sink nodes mobility model is described as pathway mobility. Here we considered the circular pathway mobility. In that the sink nodes follow the circular path. While moving in the circular path in order to collect the data the sink nodes will be in static points or positions. Let $\mathrm{x}, \mathrm{y}$ are the coordinates of the initial point, ' $r$ ' is the radius of the circular path and the sink nodes move with a velocity, $\mathrm{v}(\mathrm{m} / \mathrm{min})$ then the next static point is obtained using the equation given below.

$$
\begin{aligned}
& x(\text { new })=x+r \cos (\alpha) \\
& y(n e w)=y+r \sin (\alpha)
\end{aligned} \text { and }
$$

Here $\mathrm{x}($ new $), \mathrm{y}($ new) coordinate indicate the next static point and ' $\alpha$ ' is the suspended angle between previous and new position. Figure 4 shows the circular path mobility with 8 static positions and also with 16 static positions. This indicates that during mobility the sink node remains static for eight or sixteen times per cycle according to our scenario.
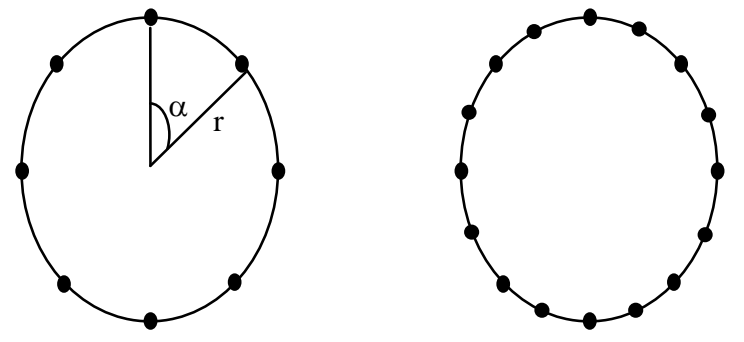

Figure 4: Circular path mobility with 8 and 16 points

\subsection{Impact of wind mobility}

This mobility model is the proposed model to enhance the lifetime of the network. Wind mobility is nothing but the mobility in which direction of movement is based on the eight wind directions. The sink nodes follow the eight directions one by one in order. It will look like octagon when the length of each move is equal. Also it is similar to circular mobility but gives notable difference in energy spent for mobility and location update. Here we considered the equi-sided octagon in order to model the wind mobility in each wind direction which is shown in figure 5 .
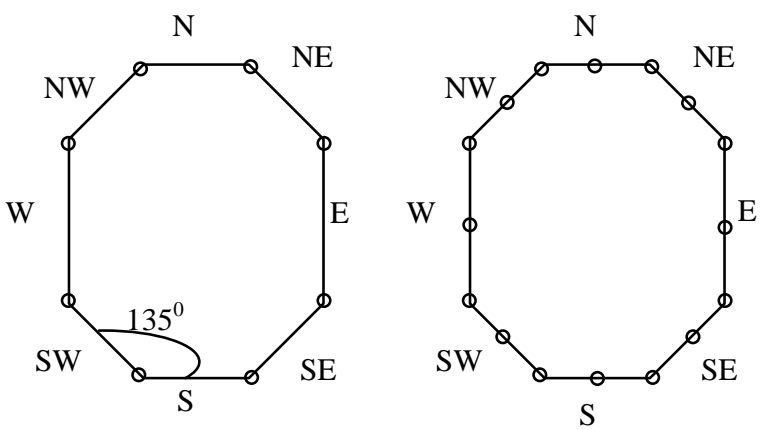

Figure 5: Wind mobility with 8 and 16 points

\subsection{Comparison of different mobility models}

The energy spent to update the locations [5] of the sink nodes every time is determined and cost for one full cycle (in our scenario either 8 points or 16 points) is calculated. For random way point mobility the energy spent is given by,

$$
E_{n}=\frac{N}{n} h T v \sum_{i=1}^{n}\left(\frac{R_{i}^{2} \pi \beta}{L(\operatorname{rand}(v, d))}+\frac{1}{R_{i} \alpha}\right)
$$

For circular mobility the energy spent is given by,

$$
E_{n}=\frac{N}{n} h T v \sum_{i=1}^{n}\left(\frac{R_{i}^{2} \beta}{r^{2} L}+\frac{1}{R_{i} \alpha}\right)
$$

For wind mobility the energy spent is given by,

$$
E_{n}=\frac{N}{n} h T v \sum_{i=1}^{n}\left(\frac{R_{i}^{2} \pi \beta}{L a^{2}(2+2 \sqrt{2})}+\frac{1}{R_{i} \alpha}\right)
$$

Where ' $\mathrm{N}$ ' is the number of static sensor nodes, ' $\mathrm{n}$ ' is the number of mobile sink nodes, ' $\mathrm{v}$ ' is the velocity of the sink nodes, ' $r$ ' is the radius of coverage area of the sink node, and ' $L$ ' is the side length of the sensing field.

\subsection{Conditions Considered}

For our simulations we considered the square shape of sensing field with homogenous static sensor nodes. Sink nodes are considered as mobile nodes and are homogenous with higher energy and memory than normal sensor nodes. Sink nodes are considered without sensing unit. Initial energy is taken as $1 \mathrm{~J}$. Total numbers of sensor nodes are 1000 and sink nodes are 10.

\section{SIMULATION RESULTS}

Based on the mathematical equations the modeling is done with the matlab and the results are shown in the figures. Figure 6 shows the random way point mobility scenario. Area coverage by the sink nodes with respect to time can be understood from the same output graph. 


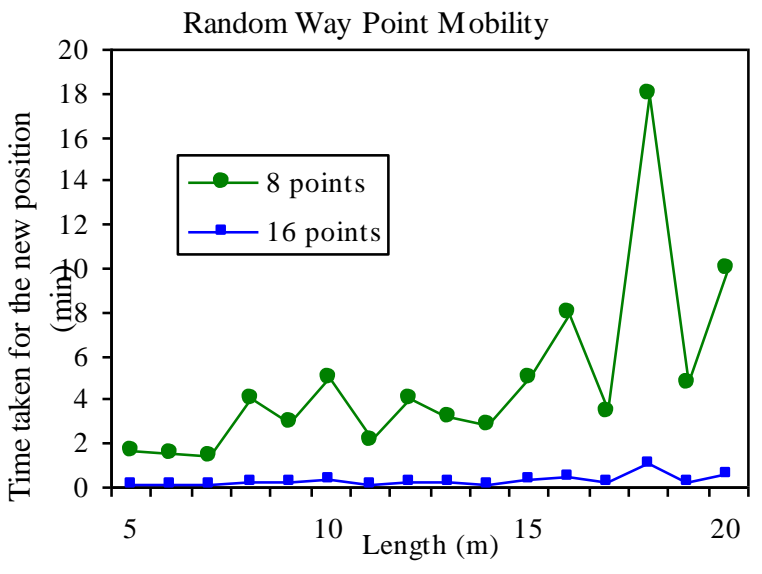

Figure 6: Time taken by the sink node to reach new positions in random way point mobility

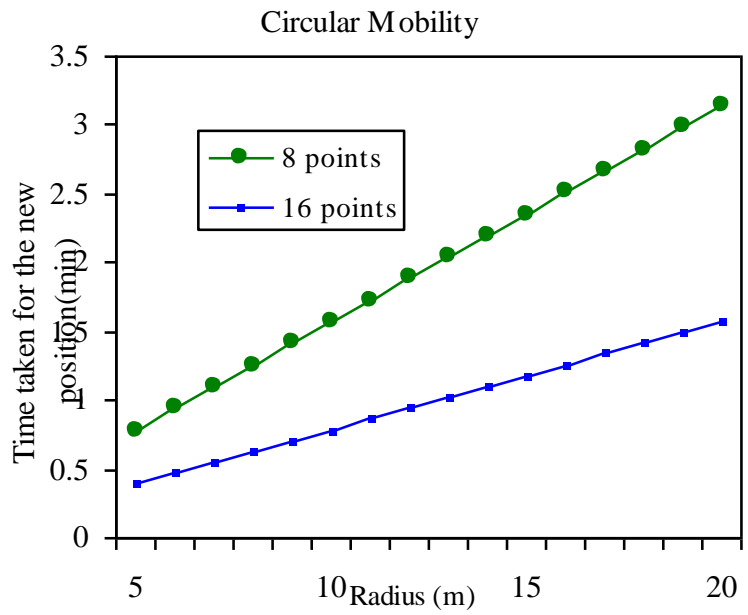

Figure 7: Time taken by the sink node to reach new positions in Circular mobility with velocity $5 \mathrm{~m} / \mathrm{min}$

Circular Mobility

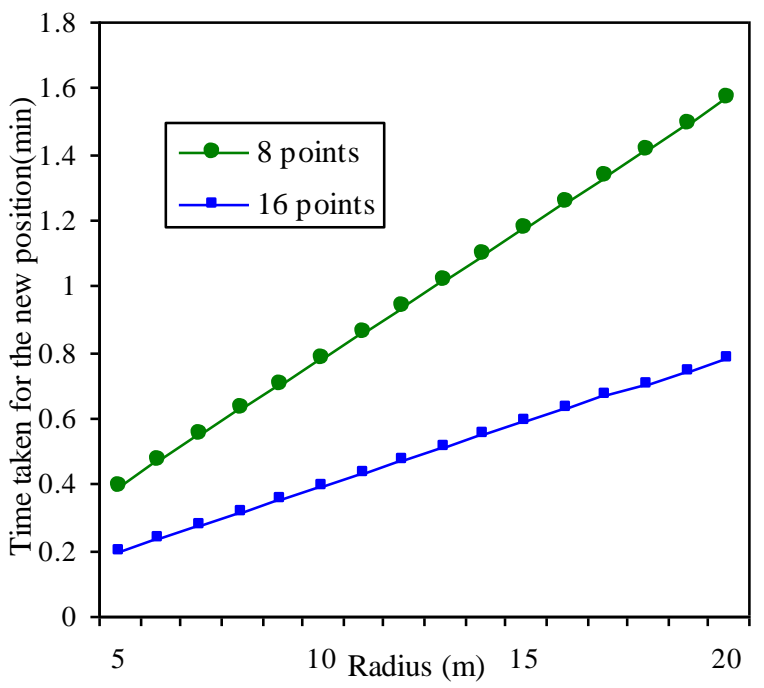

Figure 8: Time taken by the sink node to reach new positions in circular mobility with velocity $10 \mathrm{~m} / \mathrm{min}$
Similarly figure 7 and figure 8 shows about the circular mobility scenario with different velocity of sink nodes. Figure 9and figure 10 shows about the wind mobility scenario with different velocity of sink nodes.

\section{Wind Mobility}

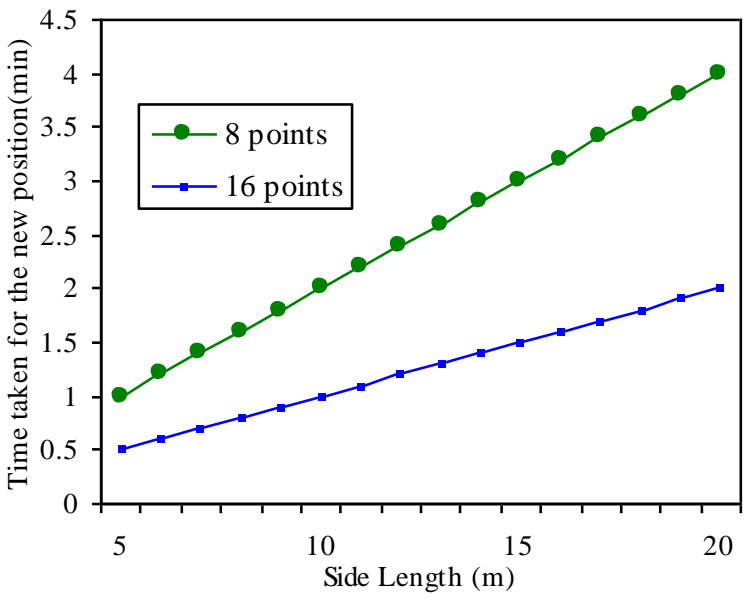

Figure 9: Time taken by the sink node to reach new positions in wind mobility with velocity $5 \mathrm{~m} / \mathrm{min}$

Wind Mobility

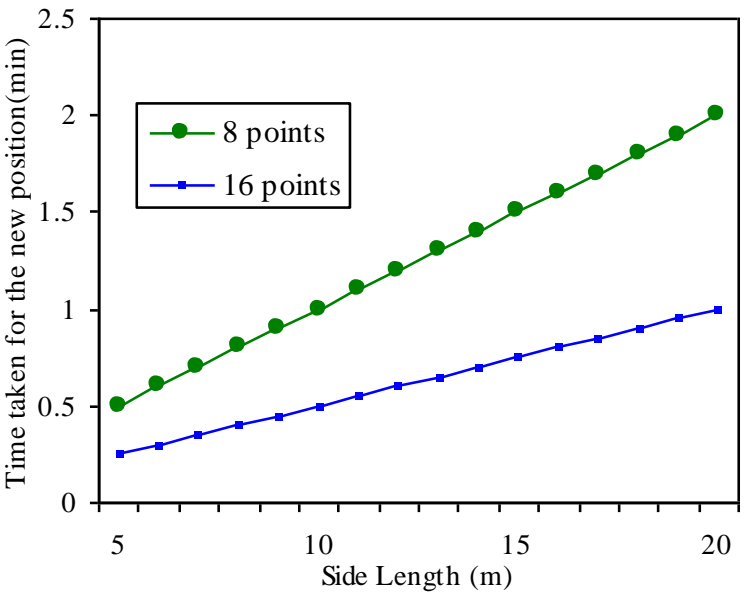

Figure 10: Time taken by the sink node to reach new positions in wind mobility with velocity $10 \mathrm{~m} / \mathrm{min}$

Figure 10 shows the energy remained after updating the sinks' new location to all the nodes for one complete cycle. Here one cycle indicates only the eight static positions. So the sink nodes will collect the data only during this static period. From the figure 10 it is observed that for wind mobility the energy spent for updating sinks' location is less compared to circular path mobility and random way point mobility. Thus the energy saved from the updating cost can be effectively utilized for aggregation or routing since wireless sensor network is a energy constraint network. 
Comparison of Mobility Models

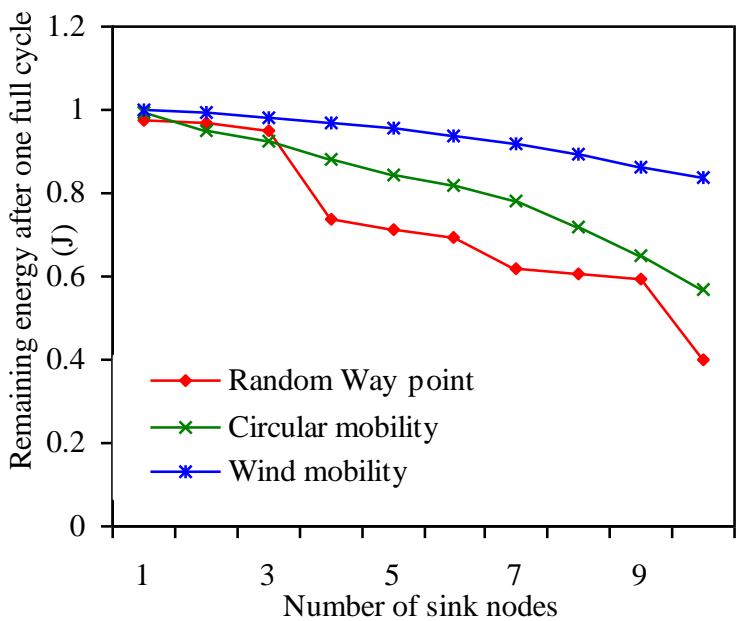

Figure 11: Remaining energy after the movement of eight positions

\section{CONCLUSION}

From the simulation results it is observed that WIND mobility model applied on the sink collects more data with minimum time than that of random way point and geographic based circular pathway mobility. In this model energy of the total network is saved which in turn increase the lifetime of the network. Increase in lifetime gives more collection of data. In future apart from pathway and random models, WIND mobility model can be optimized with a spatial based mobility models.

\section{REFERENCES}

[1] Jamal Al-Karaki, Ahmed E.kamal, "Routing Techniques in Wireless Sensor Networks", IEEE Wireless Communications. Dec.2004.

[2] Jun Luo, Jean-Pierre Hubaux, "Joint Mobility and Routing for lifetime Elongation in Wireless Sensor Networks", IEEE INFOCOM, 2005.

[3] Guojan Wang, Tian Wang, et.al., "Local Update Routing Protocol in Wireless Sensor Networks with Mobile Sinks", IEEE Communications Society, 2007.

[4] Karim Seada, Ahmed Helmy, "Efficient Geocasting over Perfect Delivery in Wireless Networks", IEEE Communications Society, WCNC 2004.
[5] M.S.Godwin Premi, K.S.Shaji, "MMS Routing for Wireless Sensor Networks", IEEE Computer Society, Feb.2010.

[6] M.S.Godwin Premi, K.S.Shaji, "MMS Routing to Enhance Lifetime in Wireless Sensor Networks", Conference Proceedings of Wireless Communication and Sensor Networks, Dec.2009.

[7] M.S.Godwin Premi, K.S.Shaji, "Router Performance with AoI and RoI Routing for Wireless Sensor Networks", IEEE International Conference on Communication Control and Computing Technologies, Oct.2010.

[8] Soyturk, M. Altilar, T., "A Novel Stateless EnergyEfficient Routing Algorithm for Large-Scale Wireless Sensor Networks with Multiple Sinks", IEEE Annual Wireless and Microwave Technology Conference, 2006. WAMICON '06.

[9] Fan Bai, Ahmed Helmy, "A survey of mobility models in Wireless Adhoc Networks".

[10] Nicholas Cooper, Natarajan, Meghanathan, "Impact of mobility models on multipath routing in mobile adhoc networks", International Journal of Computer Networks \& Communications (IJCNC), Vol. 2, No.1, January 2010.

[11] Mohd Izuan Mohd Saad, Zuriati Ahmad Zukarnain, "Performance Analysis of Random-Based Mobility models in MANET Routing Protocol", European Journal of Scientific Research, Vol.32 No.4 (2009), pp.444-454.

[12] Institut Eur'ecom, Department of Mobile Communications, "Mobility Models for Vehicular Ad Hoc Networks: A Survey and Taxonomy", Research Report RR-06-168, March 26th, 2007.

[13] Rainer Baumann, Simon Heimlicher, Mario Strasser, Andreas Weibel, "A Survey on Routing Metrics", TIK Report 262,Computer Engineering and Networks Laboratory, ETH-Zentrum, Switzerland, February 10, 2007.

[14] Hannes Frey, Stefan R"uhrup, and Ivan Stojmenovi'c, "Routing in Wireless Sensor Networks", Guide to Wireless Sensor Networks, Computer Communications and Networks, Springer-Verlag London Limited, 2009.

[15] Sameera Poduri, Gaurav S. Sukhatme, "Constrained Coverage for Mobile Sensor Networks", IEEE International Conference on Robotics and Automation, pages 165-172, April 26-May 1, 2004, LA, USA. 